\title{
A Dynamic Transmission Line Model to Describe the Potential Dependence of Double Layer Capacitances in Cyclic Voltammetry
}

\author{
Maximilian Schalenbach*a, Y. Emre Durmus ${ }^{\text {a }}$, Hermann Tempel ${ }^{\text {a }}$, Hans Kungl ${ }^{\text {a }}$ and Rüdiger-A. Eichel ${ }^{\text {a }}$ \\ a Fundamental Electrochemistry (IEK-9), Institute of Energy and Climate Research, Forschungszentrum Jülich \\ GmbH, 52425 Jülich, Germany, \\ * Corresponding author: m.schalenbach@fz-juelich.de
}

This supporting information discusses the differential equations of the dynamic transmission line model, the resistances and capacitances of the ladder network, its response in the time and frequency domain, and the impact of the ladder parameters on the response. Moreover, the cyclic voltammetry $(\mathrm{CV})$ response of the transmission line model is compared to a previously reported Fourier transformation model. Finally, the computer codes to reproduce the presented results are presented.

\section{Content}

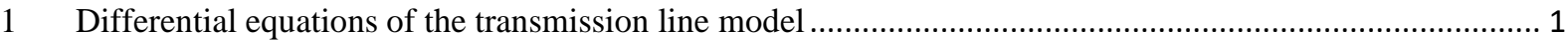

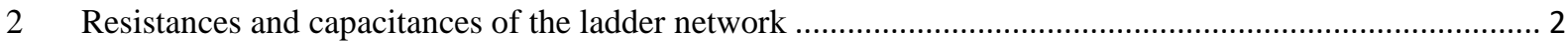

3 Validity of the transmission line model in the frequency domain ...................................................... 3

Impedance derived from time domain modeling ...................................................................... 4

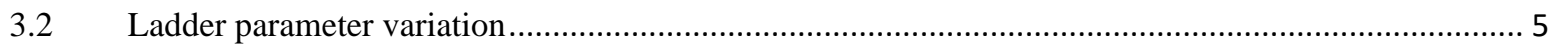

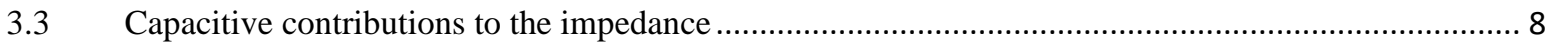

$4 \quad$ Transmission line model $v s$. Fourier transformation model …........................................................ 9

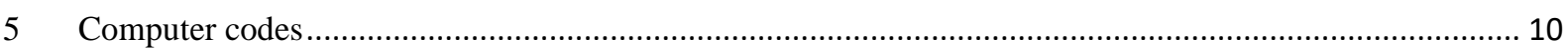

$5.1 \quad$ Polynomial fit to potential dependent CPE parameters ....................................................... 10

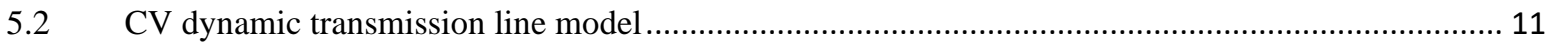

\section{Differential equations of the transmission line model}

In the following, the set of differential equations that describes the transmission line model is derived on the basis of Kirchhoff's circuit laws. To follow and understand the derivation of the equations it is recommended to consider the equivalent circuit in Figure 1B of the article. The triangular waveform of the potential variation $U_{\mathrm{pv}}(t)$ during cyclic voltammetry is described by

$$
U_{\mathrm{pv}}(t)=U_{\mathrm{start}}+v \mathrm{t}
$$

for the up-scan and

$$
U_{\mathrm{pv}}(t)=U_{\text {start }}+\frac{v \mathrm{t}_{\mathrm{per}}}{2}-v\left(\mathrm{t}-\frac{\mathrm{t}_{\mathrm{per}}}{2}\right)
$$

for the down scan, where $U_{\text {start }}$ denotes the starting potential, $v$ the scan rate and $t_{\text {per }}$ the time for one excitation period.

The equation $Q=C U$ relates charge $Q$, capacitance $C$ and voltage $U$. Taking the derivative in time leads to a linear differential equation in the form of

$$
\frac{\mathrm{d}}{\mathrm{d} t} U_{C_{k}}=\frac{I_{C_{k}}}{C_{k}}
$$

where $C_{k}$ denotes the k-th capacitor in the ladder, $U_{C_{k}}$ its voltage drop and $I_{C_{k}}$ the current that goes through this capacitor. The latter step of the ladder is denoted by the subscript $L$. 
The current through the $0^{\text {th }}$ element of the transmission line is calculated by:

$$
U_{\mathrm{pv}}=U_{0}+R_{0} I_{R_{0}}
$$

By taking the derivative in time and by replacing the voltage derivative by equation $\mathrm{S} 3$, the first differential equation to describe the response is derived:

$$
\frac{\mathrm{d}}{\mathrm{d} t} I_{R_{0}}=v-\frac{I_{C_{0}}}{C_{0}}=v-\frac{I_{R_{0}}-I_{R_{1}}}{C_{0}}
$$

In this equation, the current through the 0-th capacitor equals the difference of the current through the surrounding resistors: $I_{C_{0}}=I_{R_{0}}-I_{R_{1}}$. In addition to this first differential equation, $L$ differential equations are required to model each current through the other resistors in the transmission line.

After calculating the differential equation for $I_{R_{0}}$, the equation of $I_{R_{1}}$ is calculated next. According to Ohms law, the voltage drop between two steps in the transmission line equals the voltage drop at the corresponding connecting resistor:

$$
U_{0}-U_{1}=R_{1} I_{R_{1}}
$$

With respect to equation S5, the time difference of the voltage difference is also related to the currents through the capacitors:

$$
\frac{\mathrm{d}}{\mathrm{d} t}\left(U_{0}-U_{1}\right)=\frac{I_{C_{0}}}{C_{0}}-\frac{I_{C_{1}}}{C_{1}}
$$

Taking derivative of equation S6 and combining it with equation S7 leads to

$$
\frac{\mathrm{d}}{\mathrm{d} t} I_{R_{1}}=\frac{1}{R_{1}}\left(\frac{I_{R_{0}}-I_{R_{1}}}{C_{0}}-\frac{I_{R_{1}}-I_{R_{2}}}{C_{1}}\right)
$$

where the capacitive currents are exchanged again by the difference of the surrounding resistive currents. The general form of this equation for the $k$-th resistor in the ladder network is:

$$
\frac{\mathrm{d}}{\mathrm{d} t} I_{R_{k}}=\frac{1}{R_{k}}\left(\frac{I_{R_{k-1}}-I_{R_{k}}}{C_{k-1}}-\frac{I_{R_{k}}-I_{R_{k+1}}}{C_{k}}\right)
$$

The last step of the ladder network $(k=L)$ resembles a special case, as a following link is missing, so that the differential equation changes to:

$$
\frac{\mathrm{d}}{\mathrm{d} t} I_{R_{L}}=\frac{1}{R_{L}}\left(\frac{I_{R_{L-1}}-I_{R_{L}}}{C_{L-1}}-\frac{I_{R_{L}}}{C_{L}}\right)
$$

Using the Euler forward approach, the differential equations above were discretized. In brief, the Euler forward method describes a derivative by

$$
\frac{d y}{d t}=\frac{y_{j+1}-y_{j}}{\Delta t}
$$

where $\Delta t$ denotes the time increment and $j$ the $j$-th step of the numerical simulation. A differential equation of the form $d y / d t=f(x)$ is discretized by using the Euler forward method to

$$
y_{j+1}=\Delta t \cdot f\left(x_{j}\right)+y_{j}
$$

from which the solution $y_{j+1}$ for the next time increment can be derived. The detailed implementation in the form of the source code is given below.

\section{Resistances and capacitances of the ladder network}

Wang ${ }^{1}$ reviewed the equations (stated in the main text) to calculate the transmission line ladder network from constant phase element (CPE) parameters. Figure S1 shows the calculated resistances and capacitances of the transmission line ladder network using the CPE parameters of $\xi=95533$ and $n=0.79$, which here serve as exemplarily values. The discretization factor $h$ was varied with values of $0.001,0.01,0.1$ and 1 . The resistances increase towards higher ladder steps and higher values of $h$ whereas the capacitances decrease. 

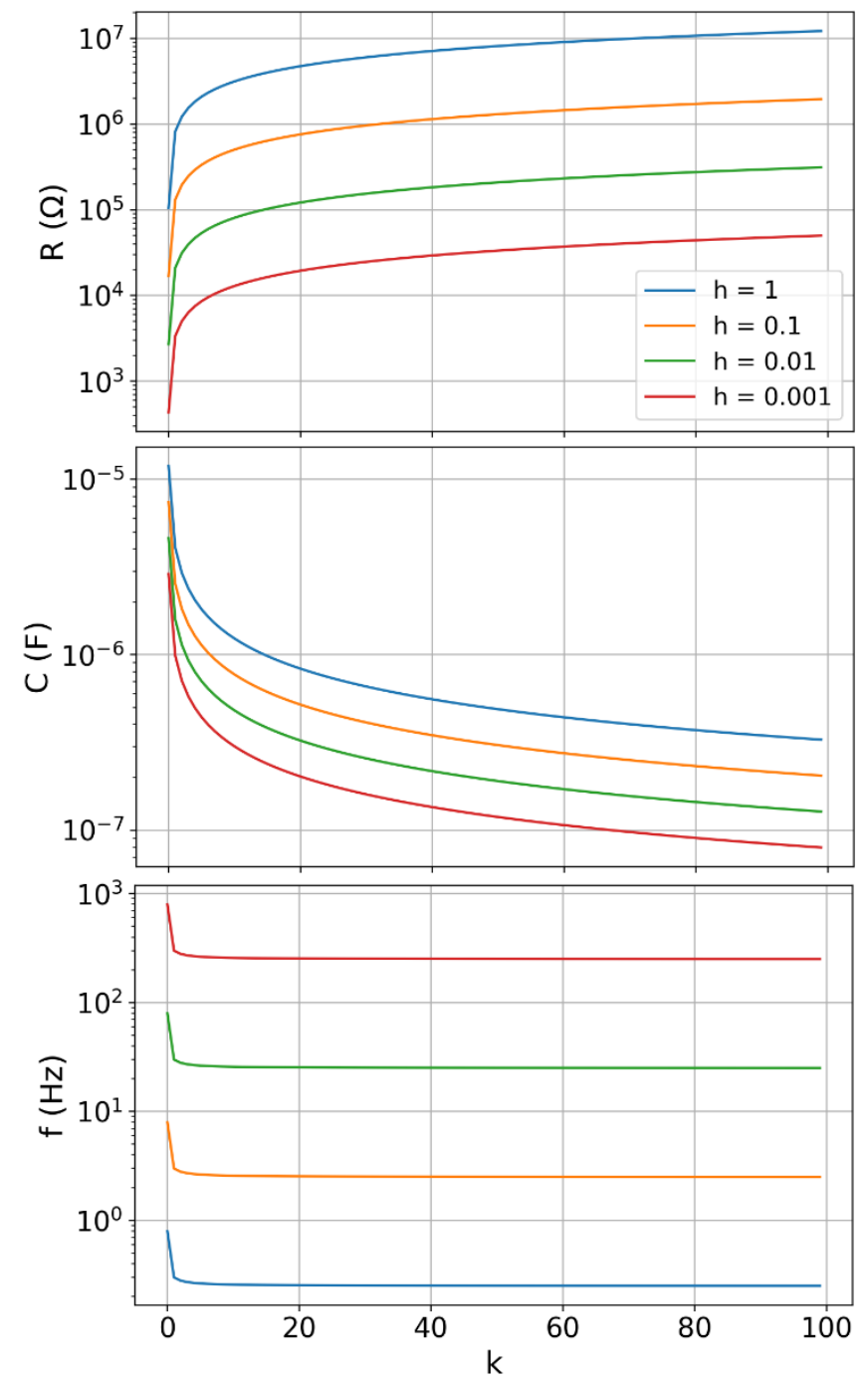

Figure S1: Resistance, capacitance and relaxation frequencies of the $k$-th steps in the ladder network under variation of the ladder discretization constant $h$

Each of the $k$-th resistor and capacitor pair have a characteristic time constant in their response, which later becomes an important property to understand the relaxation processes of the transmission line network responses to potential variations. The relaxation frequency for each pair of resistor and capacitor can be determined to ${ }^{2}$ :

$$
f_{k}=\frac{1}{2 \pi R_{k} C_{k}}
$$

Figure S1 also shows these relaxation frequencies for each ladder step. The relaxation frequencies of the first three elements of the ladder are significantly higher than that of the following ladder steps which approach a constant value. The relaxation frequencies change proportionally to the factor $h$. When the potential variation or some of its dominant higher harmonics ${ }^{3}$ are in the same order of magnitude as the relaxation frequency of the transmission line model, the current response is damped by the relaxation and thus does not represent an adequate formulation of the CPE as further evaluated below.

\section{Validity of the transmission line model in the frequency domain}

In impedance spectroscopy, a CPE reacts to the sinusoidal potential variation with a sinusoidal current that is characterized by two parameters, a phase shift and amplitude. In contrast, the shark fin type response of the CPE to the triangular potential variation used in $\mathrm{CV}$ is more complex and more difficult to describe ${ }^{3}$. The definition of the CPE in impedance spectroscopy allows to calculate analytical solutions in the frequency domain. When the numerically calculated response of the transmission line model (that represents the CPE) to sinusoidal potential variations is transferred from the time domain to the frequency domain, it can be directly compared to the 
analytically calculated impedance. In this section, the response is again considered for the CPE parameters of $\xi=$ 95533 and $n=0.79$ while the electrolyte resistance is neglected.

\subsection{Impedance derived from time domain modeling}

In the following, the response of the numerical transmission line model to sinusoidal potential variations is translated from the time domain to the frequency domain. The potential variation of the sinusoidal excitation in the time domain $U_{\mathrm{pv}}(t)$ is described by

$$
U_{\mathrm{pv}}(t)=U_{\mathrm{amp}} \sin (\omega \mathrm{t})
$$

where $U_{\mathrm{amp}}$ denotes the peak-to-peak amplitude. The impedance is characterized by its amplitude $|Z|$ and phase angle $\phi$. The amplitude can be calculated by the maximum of the potential $U_{\max }$ divided by the maximum of the current $I_{\max }$ :

$$
|Z|=\frac{U_{\max }}{I_{\max }}
$$

In the time domain, the phase angle $\phi$ in radiant can be calculated by characteristic time differences as described by

$$
\phi=2 \pi \frac{t_{\mathrm{cross}}-t_{\mathrm{end}}-t_{0}}{t_{\mathrm{end}}-t_{0}}
$$

where $t_{\text {cross }}$ denotes the second time the current crosses the average potential, $t_{\text {end }}$ the end time of the voltage variation period and $t_{0}$ its start time. Figure S2 exemplarily shows the response of the transmission line model to a sinusoidal potential variation in the time domain with the characteristic times $t_{0}, t_{\text {cross }}$ and $t_{\text {end }}$. Due to different starting conditions, the first period is significantly different than the following ones. The first period displays a non-linear response and cannot be characterized by a single sinusoidal function. During the following periods the system is settled with the characteristic sinusoidal response that is required for impedance calculations. Thus, the second period is used to calculate $|Z|$ and $\phi$.

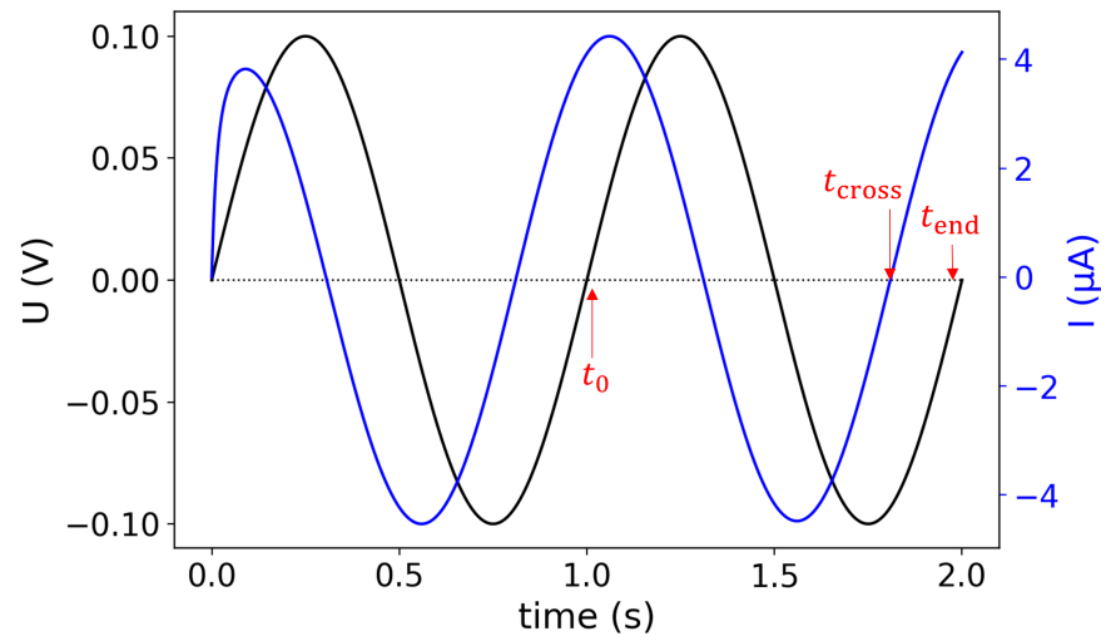

Figure S2: Numerically calculated current response (blue) of the transmission line model to a sinusoidal potential variation (black) of 1 Hz. The characteristic times $t_{0}, t_{\text {cross }}$ and $t_{\text {end }}$ are used to calculate the phase angle of the response.

Figure S3 shows the normalized currents through the individual capacitors of the ladder network for a frequency of $1 \mathrm{~Hz}$. The response of the first element is mostly capacitive $\left(\phi \approx-80^{\circ}\right)$, while the phase angle increases towards higher steps of the ladder network. The resistive share increases along the ladder network, as the current goes through more resistors before it is short circuited by a capacitor. In addition, the values of the capacitors decrease towards higher ladder steps. Furthermore, the phase angle of the 0-th resistor-capacitor pair is inherited to the 1-th resistor and so forth, so the phase angle changes along the ladder. 


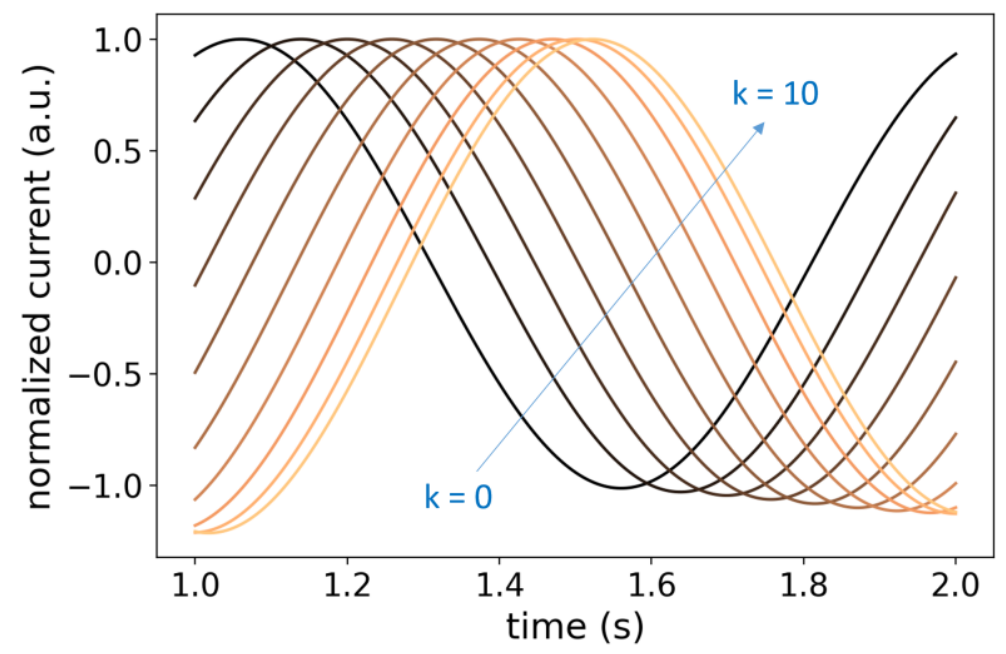

Figure S3: Normalized currents through the first ten resistors of the ladder network (calculated with the same data set of the transmission line model as that in Figure S2).

\subsection{Ladder parameter variation}

In the following, the effect of the ladder parameters (discretization constant $h$ and the ladder length $L$ ) on the responses of the transmission line model to sinusoidal potential variations is characterized.

\section{Variation of the discretization factor}

The ladder discretization factor $h$ showed an enormous impact on the resistances, capacitances and resonant frequencies of the individual ladder steps in Figure S1. Thus, when the CPE-parameters drastically change, the transmission line model must be analyzed to obtain an adequate discretization factor. To understand the validity of the transmission line model for the set of parameters in this supporting information, its response is modeled in a frequency range of approximately eight decades for a ladder discretization factor variation of $h=1,0.1,0.01$, 0.001 and a ladder size of 50 steps. Based on the response of the transmission line model in the time domain the related impedance is calculated as described above. Figure S4 displays the outcome of this parameter study, showing that the calculated impedance spectra of the different discretization factors have a similar shape with shifted frequencies due to the different relaxation times. 


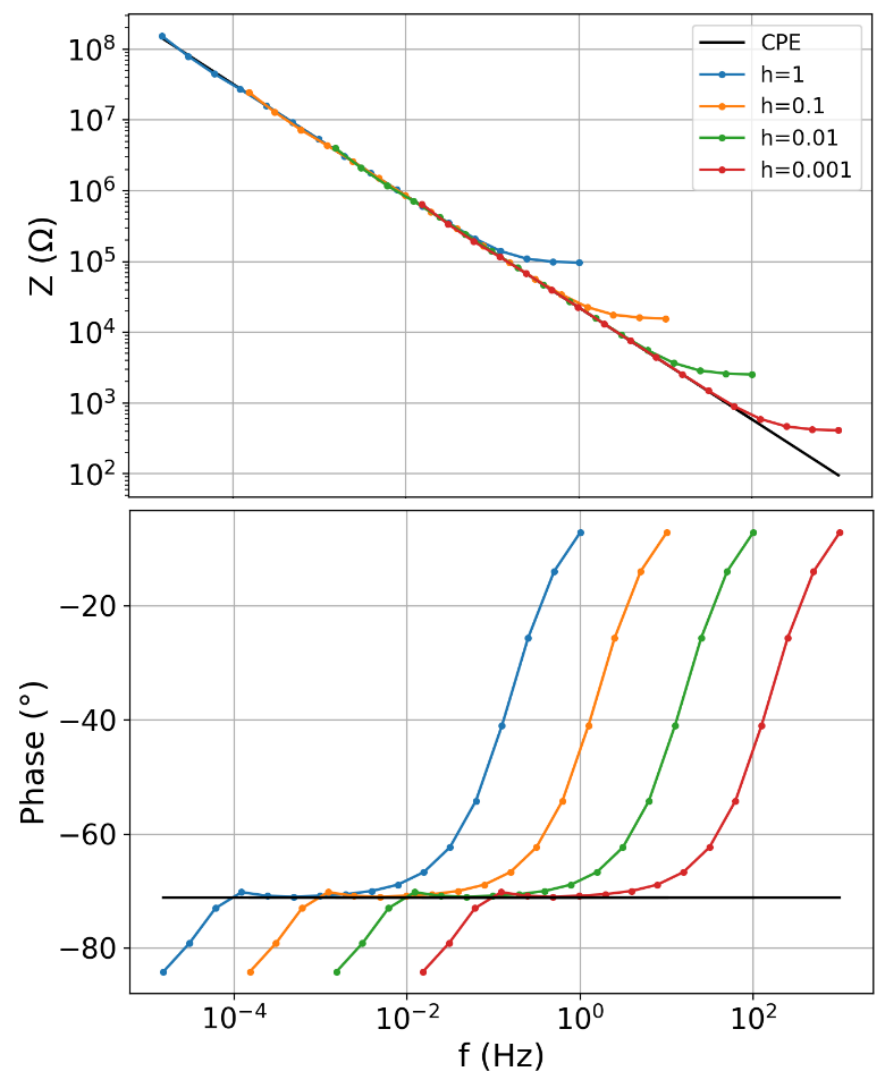

Figure S4: Model impedance. Solid black lines: Analytically calculated impedance of a CPE. Colored lines and scatter: Impedance derived from the transmission line model under a variation of the discretization factor using a ladder size of 50 steps.

With reference to Figure S1, the relaxation frequencies of the ladder network decrease reciprocally to the discretization factor (values for the lowest relaxation frequency are $0.04,0.4,4$, and $40 \mathrm{~Hz}$ for $h=1,0.1,0.01$, and 0.001). Figure S4 shows that at higher frequencies than the lowest relaxation frequency of a ladder network, the impedance $|Z|$ and phase angle are larger than that of the analytical solution. The too resistive phase angles result from the resistive contributions of the relaxation in the ladder network that increasingly dominate the response toward higher frequencies.

At lower frequencies than the lowest relaxation frequencies of the ladder networks, the impedance $|Z|$ of the transmission line model agrees with the analytically calculated data (the deviations of both cannot be seen in this depiction and will be highlighted later). The phase angle of the numerical and analytical solutions agree within one decade of the frequency before the numerical solution becomes too capacitive. As currents decrease towards lower frequencies, the currents go through all the ladder steps without being significantly damped. Thus, the negligible Ohmic drops at the resistors leads to a dominating capacitive response for which the phase angle derived from the transmission line model does not resemble the constant phase behavior anymore.

To understand the deviations from the analytical solution in more detail, Figure S5 shows the maximum currents of the individual capacitors as a function of the frequency for a ladder size of 50. At high frequencies, the current drastically decreases along the ladder. In this case the 0-th resistance dominates and leads to too resistive contributions, as the response is dominated by the RC-relaxation of the firsts steps of the ladder network and the related resistive damping. Towards low frequencies the currents decrease so that the Ohmic Drop at the beginning of the ladder decreases and the current is more equally distributed along the entire ladder network. In the considered case, the best agreement of the ladder network and the analytically calculated CPE is achieved between 0.01 to $0.2 \mathrm{~Hz}$, where the current through the 0 -th and 50-th ladder steps span between 2 and 4 order of magnitudes. In this regime, the response is neither too resistive (case of a too fast dropping current along the ladder network) nor too capacitive (too slowly dropping current and too big contributions of the last steps of the ladder network). 


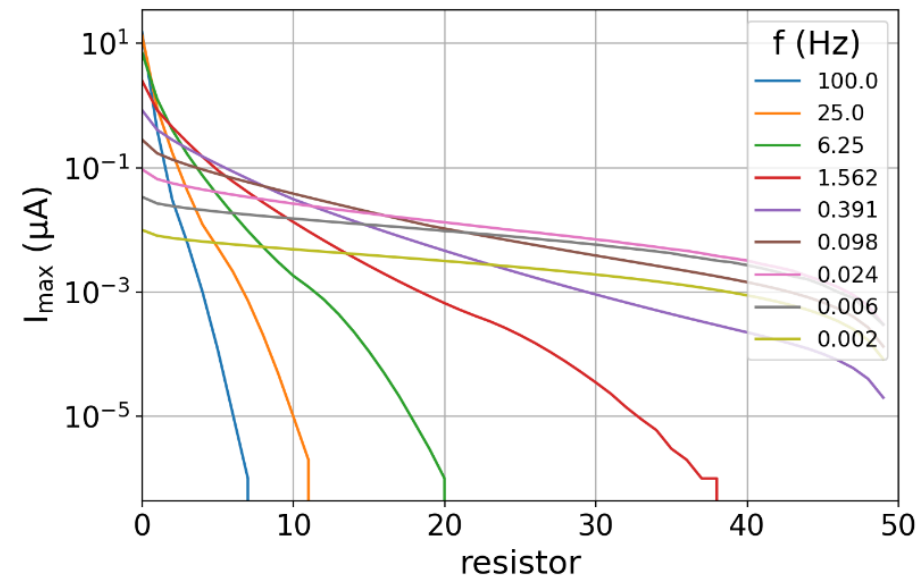

Figure S5: Amplitudes of the numerically calculated currents through the individual resistors of the ladder network as a function of a frequency variation. A discretization factor of $h=1$ and a ladder size of 50 steps was used for these calculation.

\section{Variation of the ladder length}

Besides the discretization factor $h$, also the ladder length $L$ is an important property that characterizes the response of the ladder network. In the following, a constant discretization factor of $h=0.01$ is used while the ladder length is varied with values of 5, 10, 25, 50 and 75. Figure $\mathrm{S} 6$ shows the impedance spectra of the transmission line model for these different ladder lengths and the analytical solution of the CPE. At a ladder length of 50, the numerical modeled phase angle equals the analytical solution from approximately 0.2 to $0.01 \mathrm{~Hz}$, spanning over more than one decade in the frequency. Towards smaller ladder networks, the frequency range with agreement of the transmission line model and the analytical solution decreases. The physical reason for the decreasing phase angles at small frequencies towards smaller ladder sizes can be found in the above described capacitive dominance. The smaller the ladder network, the more dominant the contributions of the capacitances become while the contributions of the resistances are getting smaller. The longer the ladder network, the more balanced is the mix of resistive and capacitive components as the capacitances decrease and the resistances increase along the ladder steps. Accordingly, the impedance spectra calculated with longer ladder networks show better agreement with the analytically solution of the CPE. However, the longer the ladder, the more computational effort is required to calculate the response and the more unstable the code becomes. 


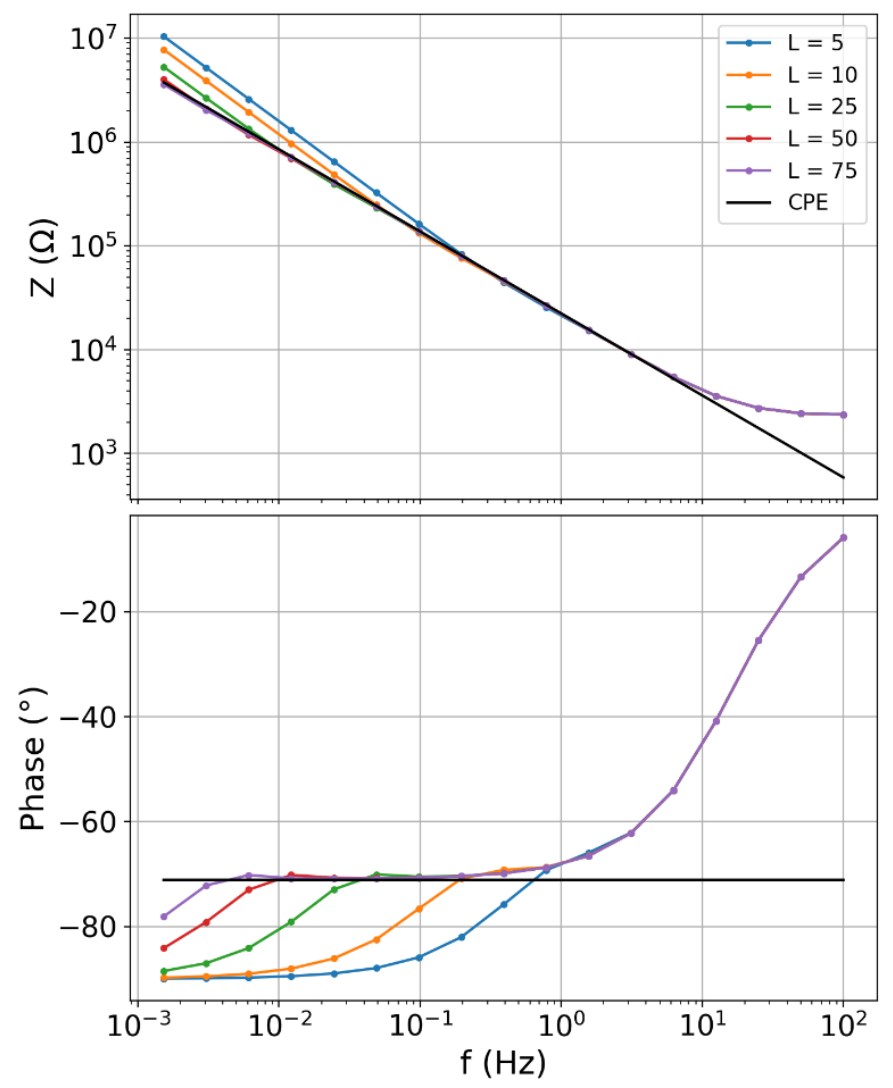

Figure S6: Colored lines: Influence of the ladder size on the impedance spectra of the transmission line model using a discretization factor of $h=0.01$. Black line: Analytically calculated response of the CPE.

\subsection{Capacitive contributions to the impedance}

The previous figures already showed deviations between the transmission line model and the analytical solution. The calculated impedance spans over several orders of magnitude in the considered frequency range, for which small differences are not visible in the graphed impedance spectra. To examine these deviations in more detail, the capacitive contributions to the response are analyzed in the following, as these are more sensitive to small deviations. These capacitive contributions to the impedance $C(\omega)$ can be derived from the impedance by

$$
C(\omega)=-\frac{1}{\omega Z^{\prime \prime}(\omega)},
$$

where $\omega$ denotes the angular frequency and $Z^{\prime \prime}(\omega)$ the imaginary part of the impedance ${ }^{2}$. The capacitive contributions to the impedance are of relevance for the overall response in $\mathrm{CV}$ as they directly influence the magnitude of the current response.

Figure S7 shows $C(\omega)$ as a function of the ladder length for $h=0.01$, calculated for the numerical transmission line model, the analytical impedance of the transmission line and the analytical result of the CPE. The $C(\omega)$ of the transmission line that is calculated analytically in the frequency domain with the impedance calculus agrees with the numerical model in the time domain, showing that both approaches lead to nearly the same result. Similar to Figure S6, the frequency range where the data of the ladder network and the analytically calculated CPE agree increases with the ladder length. Despite the data in Figure S6 for a ladder size of 50 steps only agreed within one decade of the frequency, the capacitive contributions to the impedance agree over more than 3 orders of magnitude, showing that the capacitive contributions to the impedance are more robust in the transmission line model than the phase angle. 


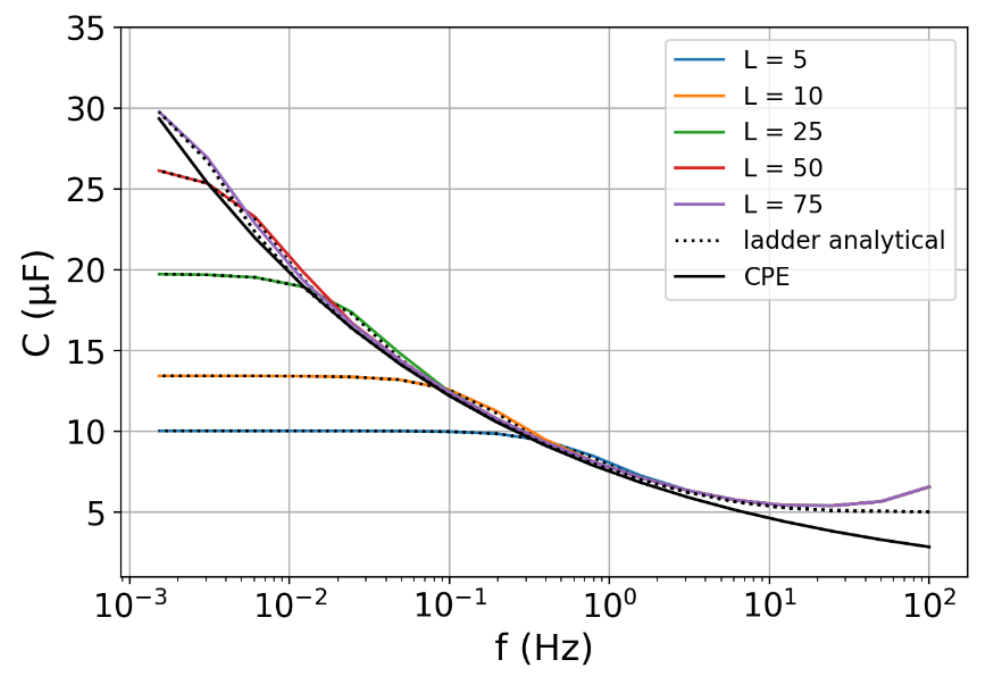

Figure S7: Capacitive contributions to the impedance calculated by the numerical transmission line model in the time domain (colored lines), analytical solutions of the transmission lines in the frequency domain (dotted black lines) and an analytical solution of the CPE. The solutions calculated with the transmission line use for a discretization factor of $h=0.01$, while the lengths of the ladder networks were varied from 5 to 75 steps.

\section{Transmission line model vs. Fourier transformation model}

The aim of this section is to show that the transmission line model enables an adequate description of CV data. A previous study modeled the CV responses of CPEs on the basis of a Fourier transformation model, which uses the impedance calculus in the frequency domain ${ }^{3}$. The results of both models are compared in Figure S8 for a scan rate of $0.1 \mathrm{~V} / \mathrm{s}$, a voltage range from 0.2 to $1 \mathrm{~V}$, the same CPE parameters as above, discretization factor of $h=$ 0.01 and a ladder network length of $L=50$.

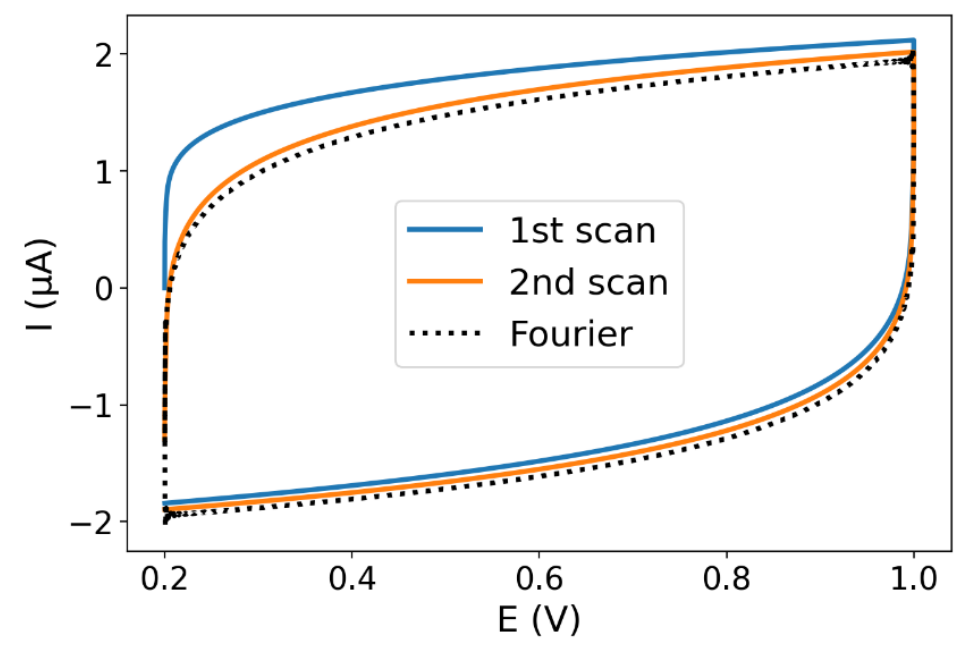

Figure S8: CV response calculated with the transmission line model (colored lines) and a Fourier transformation model (dotted line).

The frequency domain based model does not allow to distinguish between different scans of the CV response and thus represents the data after an infinite amount of scans. In contrast, in the time domain, the response of every scan is slightly different, as it starts from different initial currents. However, already during the second scan the transmission line model and the Fourier transformation model show good agreement, showing that the impact of the different starting conditions are already for the second scan small. The agreement with the data of the Fourier transformation model shows, that the ladder parameterization (in the form of $h$ and $L$ ) is neither too capacitive nor too resistive for the considered CPE parameters ( $\xi$ and $n$ ). 


\section{Computer codes}

All codes were calculated on a personal computer and do not require advanced hardware. The codes to fit the impedance spectra were reported previously ${ }^{2}$.

\subsection{Polynomial fit to potential dependent CPE parameters}

The following code is used for a polynomial fit of arrays to the CPE parameters that were previously fitted to individual impedance spectra.

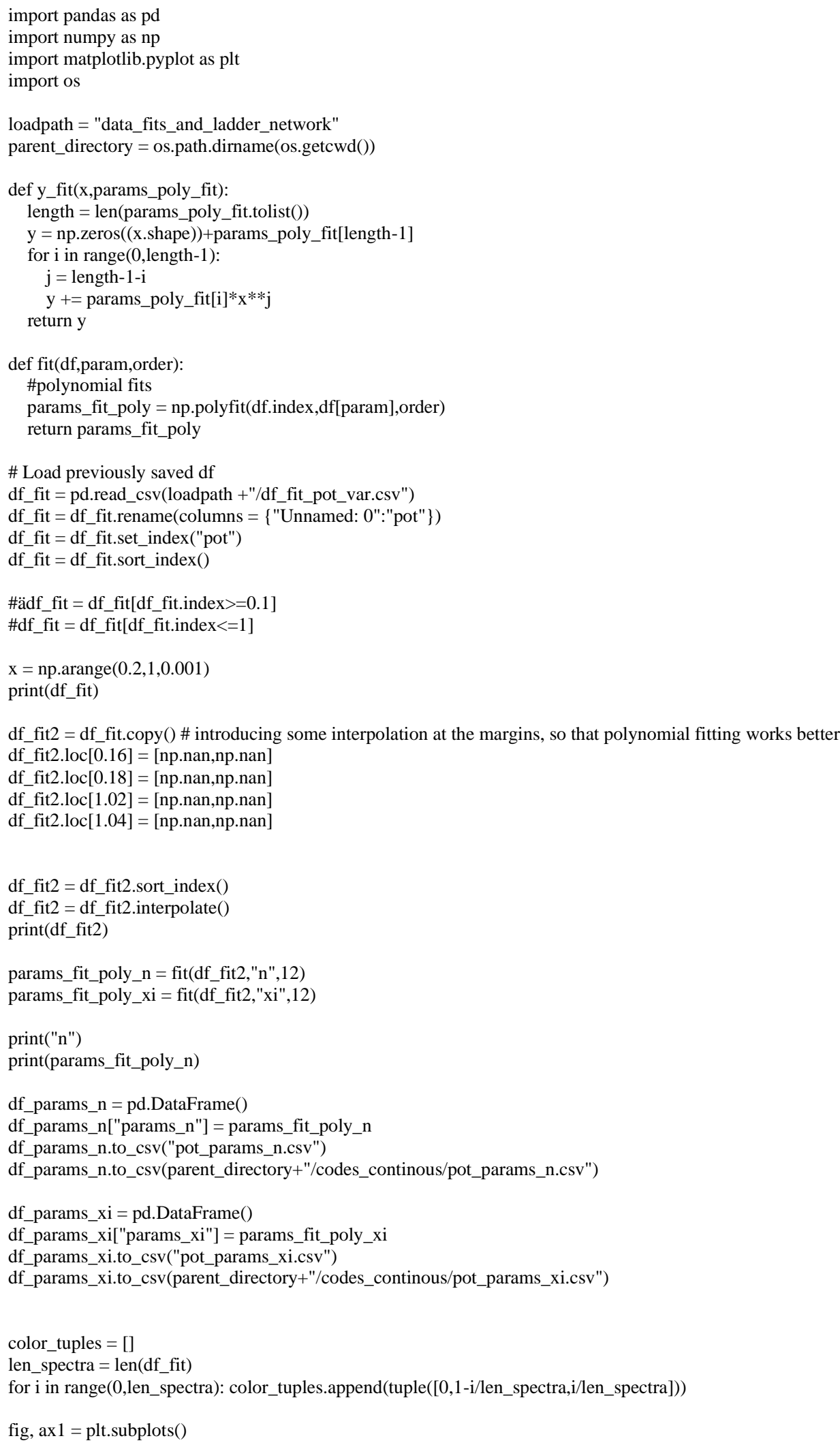


color $=$ 'tab:red'

ax1.set_xlabel('E (V vs RHE)')

ax1.set_ylabel(r'\$xi\$', color='k')

\#ax1.plot(df_fit.index, df_fit.xi, 'k:')

ax1.plot(x,y_fit(x,params_fit_poly_xi),'k:')

ax1.tick_params(axis='y', labelcolor='k')

for $\mathrm{i}$ in range(len(df_fit.index)):

ax1.scatter(list(df_fit.index)[i],df_fit.at[list(df_fit.index)[i],"xi"],marker = "^",s=100,color = color_tuples[i])

y_spread = abs(df_fit.xi.max()-df_fit.xi.min())

ax1.arrow(list(df_fit.index)[6],df_fit.at[list(df_fit.index)[6],"xi"],-0.1,0,width= y_spread/100,head_width = y_spread/25,head_length

$=0.02$, color $\left.=" k^{\prime \prime}\right)$

$\operatorname{ax} 2=\operatorname{ax} 1 . t w i n x()$ \# instantiate a second axes that shares the same $x$-axis

ax2.set_ylabel('n', color='k') \# we already handled the $x$-label with ax1

\#ax2.plot(df_fit.index, df_fit.n, color='k')

ax2.plot(x,y_fit(x,params_fit_poly_n),color="k")

ax2.tick_params(axis='y', labelcolor='k')

for $i$ in range(len(df_fit.index)):

ax2.scatter(list(df_fit.index)[i],df_fit.at[list(df_fit.index)[i],"n"],color = color_tuples[i])

y_spread $=$ abs(df_fit.n.max ()-df_fit.n.min ()$)$

ax2.arrow(list(df_fit.index)[11],df_fit.at[list(df_fit.index)[11],"n"],0.1,0,width=y_spread/100,head_width = y_spread/25,head_length

$=0.02$, color $\left.=" k^{\prime \prime}\right)$

fig.tight_layout() \# otherwise the right y-label is slightly clipped

plt.xlim(0.198,1)

plt.savefig(loadpath + "/fit_params.png", dpi = 200)

plt.show()

\subsection{CV dynamic transmission line model}

The following code shows the code used to calculate the CV responses with the dynamic transmission line model.

"'"

Created on Fri Jan 17 09:40:23 2020

@ author: m.schalenbach

"""

import sys

import os

import shutil

import pandas as pd

import numpy as np

import matplotlib.pyplot as plt

import math

import scipy.special as scs

import matplotlib

matplotlib.rc('xtick', labelsize=16)

matplotlib.rc('ytick', labelsize=16)

font $1=\{$ 'family' : 'normal',

'weight' : 'normal',

'size' : 18\}

matplotlib.rc('font', **font1)

matplotlib.rcParams["figure.figsize"] = $(7.5,5)$

parent_directory = os.path.dirname $($ os.getcwd())

sys.path.insert(0, parent_directory)

import config

import fit_poly

\# =

\# \# what to do, when algorithm instable:

\# - increase frequency

\# - increase tend (more steps and smaller h)

\# - decrease ladder (tends to make algorithm more stable but phase angle to low)

\# - increase inf_factor (for calculation of network. requires larger tend)

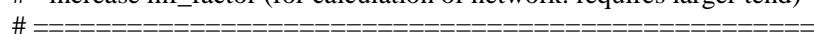

\# ========================
\# \#\#\#\# Start: Main parameters \#\#\#\#

$\#========$
\# Model option 
CV_continous $=$ True \# False: only one set of $\mathrm{n} \& \mathrm{xi}$, True: Potential dependent $\mathrm{n} \& \mathrm{xi}$ voltage_range_from_experiment $=$ True

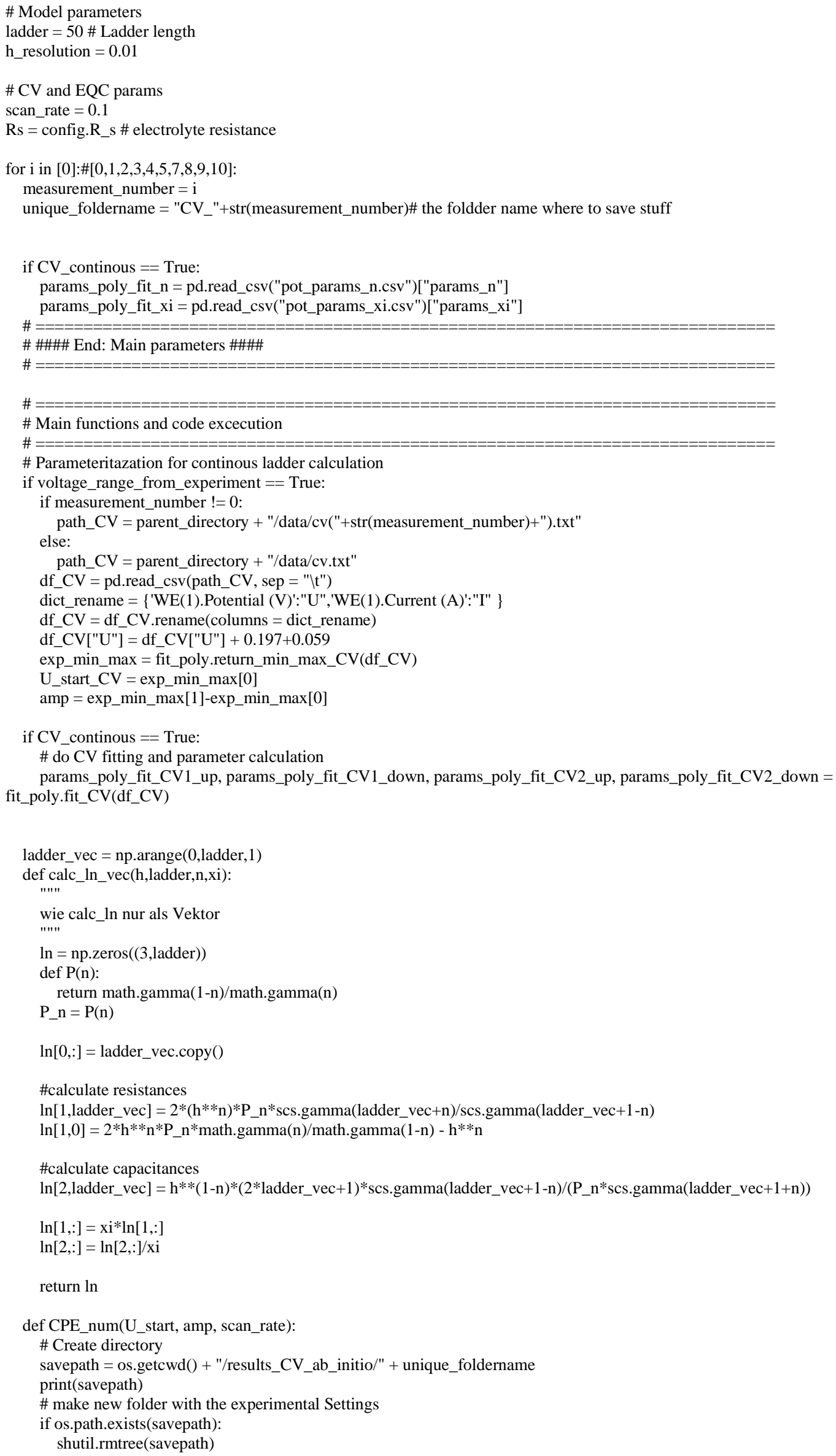


os.mkdir(savepath)

print('scan rate '+str(scan_rate))

\# ladder calculation

if CV_continous == False: \#if the ladder should be calculated only once

dummy, R, C = calc_ln_vec(h_resolution,ladder,n_static,xi_static)

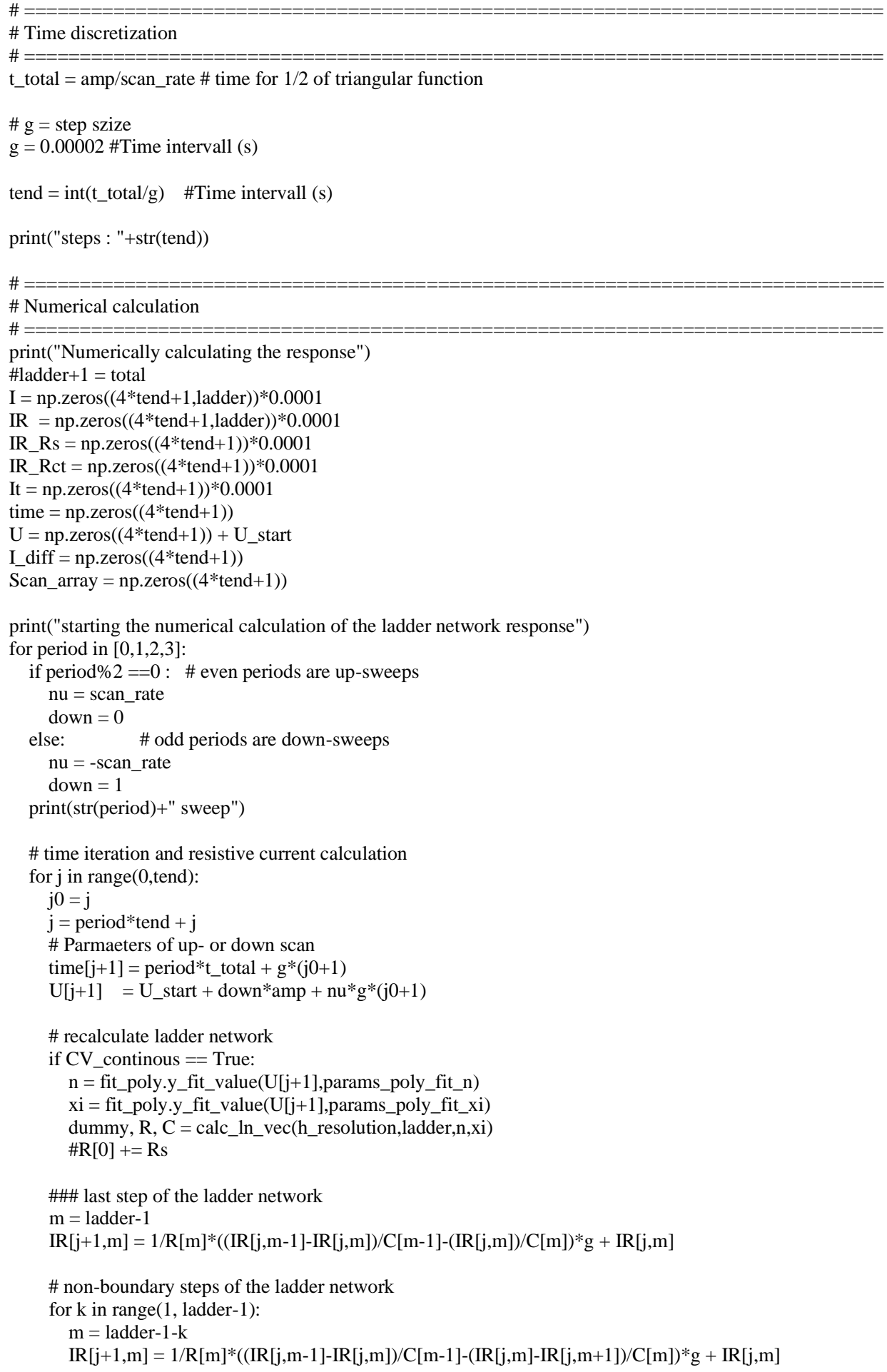




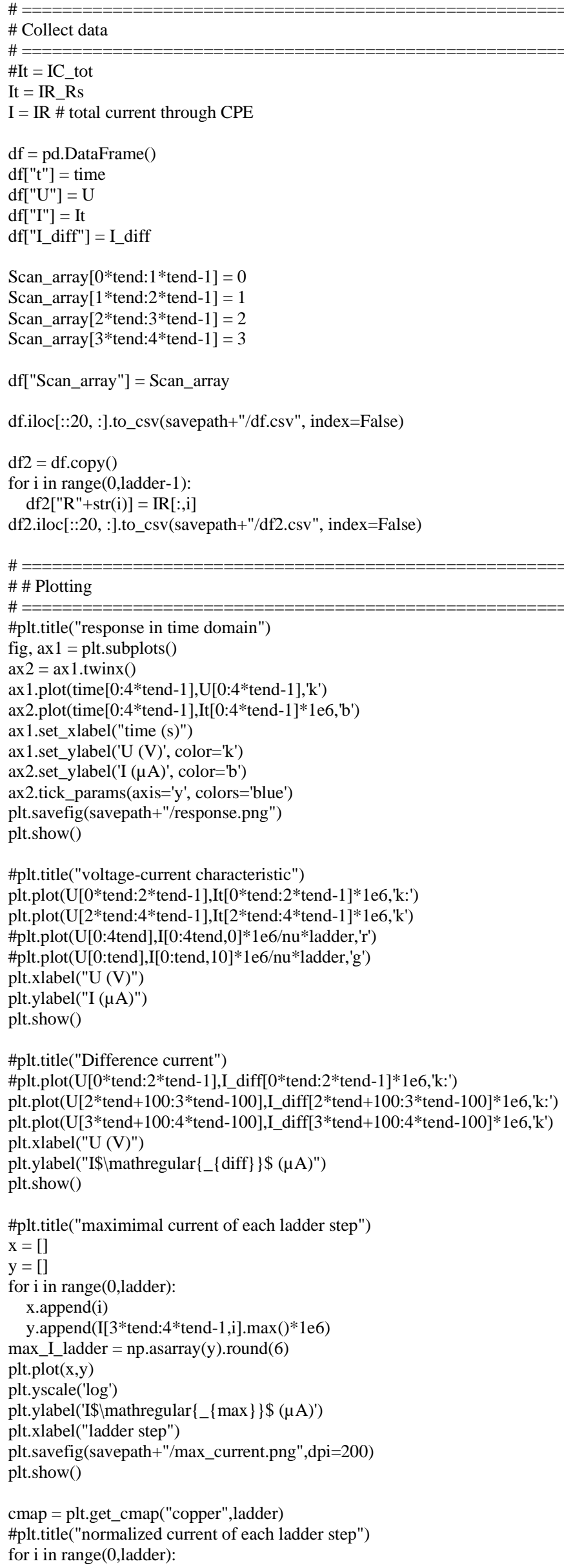




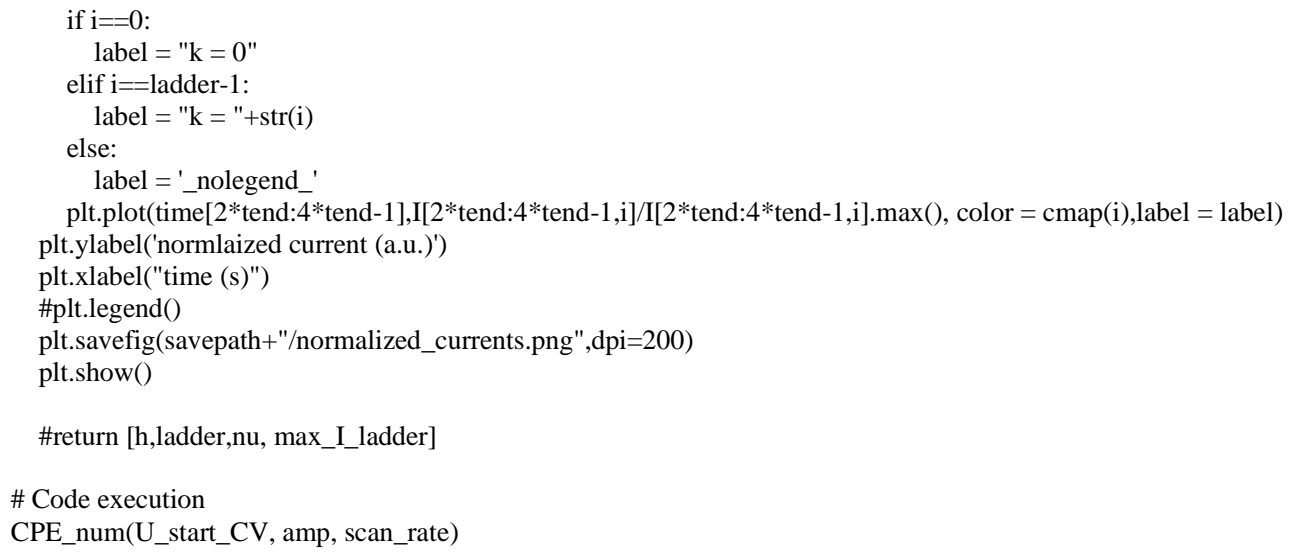

\section{References}

1 J. C. Wang, Realizations of Generalized Warburg Impedance with RC Ladder Networks and Transmission Lines, J. Electrochem. Soc., 1987, 134, 1915-1920.

2 M. Schalenbach, Y. E. Durmus, S. Robinson, H. Tempel, H. Kungl and R. Eichel, The Physicochemical Mechanisms of the Double Layer Capacitance Dispersion and Dynamics : An Impedance Analysis, Phys. Chem. C, 2021, 125, 5870-5879.

3 M. Schalenbach, Y. E. Durmus, H. Tempel, H. Kungl and R.-A. Eichel, Double Layer Capacitances Analysed with Impedance Spectroscopy and Cyclic Voltammetry: Validity and Limits of the Constant Phase Element Parameterization, Phys. Chem. Chem. Phys., 2021, 23, 21097-21105. 